\title{
Dexmedetomidine blocks thermal hyperalgesia and spinal glial activation in rat model of monoarthritis
}

\author{
Bo XU ${ }^{1}$, Wei-shi ZHANG ${ }^{1}$, Jia-le YANG ${ }^{2}$, Hua $X^{1}{ }^{1}$, Xiao-ming DENG $^{1, *}$, Yu-qiu ZHANG ${ }^{2, *}$ \\ ${ }^{1}$ Department of Anesthesiology, Changhai Hospital, The Second Military Medical University, Shanghai 200433, China; ${ }^{2}$ Institute of \\ Neurobiology, Institutes of Brain Science and State Key Laboratory of Medical Neurobiology, Fudan University, Shanghai 200032, \\ China
}

\begin{abstract}
Aim: To investigate the effect of systemic administration dexmedetomidine, a selective alpha 2 adrenergic receptor ( $\left.\alpha_{2} A R\right)$ agonist, on thermal hyperalgesia and spinal glial activation evoked by monoarthritis (MA).

Methods: MA was induced by an intra-articular injection of complete Freund's adjuvant (CFA). Thermal hyperalgesia was measured by Hargreaves' test. The spinal glial activation status was analyzed by GFAP (an astrocytic marker) and Iba-1 (a microglial marker) immunohistochemistry or immunoblotting.

Results: Unilateral intra-articular injection of CFA produced a robust glial activation of astrocytes and microglia in the spinal cord, which was associated with the development and maintenance of thermal hyperalgesia. Intraperitoneal (ip) injection of dexmedetomidine (2.5 and $10 \mu \mathrm{g} / \mathrm{kg}$ ) was repeatedly given once daily for 5 days with the first injection 60 min before intra-articular CFA. At the dose of 10 $\mu \mathrm{g} / \mathrm{kg}$, dexmedetomidine significantly attenuated MA-induced ipsilateral hyperalgesia from day 2 to day 5 . MA-induced up-regulation of GFAP expression on both sides of the spinal dorsal horn was significantly suppressed by day 5 post-MA following dexmedetomidine application, whereas MA-induced Iba-1 up-regulation was only partially suppressed.
\end{abstract}

Conclusion: Systemic dexmedetomidine inhibits the activation of spinal glia, which is possibly associated with its antihyperalgesia in monoarthritic rats.

Keywords: pain modulation; arthritis; astrocyte; spinal cord; rats; alpha 2 adrenergic receptor; dexmedetomidine

Acta Pharmacologica Sinica (2010) 31: 523-530; doi: 10.1038/aps.2010.32; published online 5 April 2010

\section{Introduction}

Arthritis is a major medical problem, with many patients experiencing chronic pain. Arthritic pain, characterized of spontaneous pain, pain in response to innocuous stimuli (allodynia) and exaggerated pain in response to noxious stimuli (hyperalgesia), is based on peripheral and central sensitization induced by inflammation of the joint ${ }^{[1]}$. It is generally believed that spinal glia (microglia and astrocytes) play a major role in the development and maintenance of central sensitization ${ }^{[2-4]}$. Central immunelike glia (microglia and astrocytes) react to peripheral inflammation such as carrageenan-induced paw inflammation ${ }^{[5]}$, complete Freund's adjuvant (CFA)-induced paw inflammation ${ }^{[6]}$ and monoarthritis ${ }^{[7-9]}$, zymosan-induced peripheral nerve inflammation ${ }^{[10]}$. Upon activation, spinal cord glia release a variety of algesic substances, particularly

\footnotetext{
* To whom correspondence should be addressed.

E-mail shxmdeng@yeah.net (XM DENG); yuqiuzhang@fudan.edu.cn (YQ ZHANG)

Received 2009-10-20 Accepted 2010-02-24
}

proinflammatory cytokines that enhance pain transmission by acting on the primary afferent neurons, spinal intrinsic neurons and spinal glia itself ${ }^{[4]}$. Disruption of spinal glial function or the action of glial products markedly block diverse exaggerated pain, suggesting that spinal glia is critically involved in the mediation of exaggerated pain evoked by inflammation or injury ${ }^{[7-9,11,12]}$.

Similarly to the clinical scenario ${ }^{[13,14]}$, dexmedetomidine, a high selective alpha 2-adrenoceptor ( $\left.\mathrm{a}_{2} \mathrm{AR}\right)$ agonist, has exhibited an significant analgesic effect in various rodent models of chronic pain, including peripheral inflammation ${ }^{[15]}$, peripheral nerve injury ${ }^{[16]}$ or spinal nerve injury ${ }^{[15]}$, and postoperative pain $^{[17]}$. Our recent study showed that intraperitoneal (ip) injection of dexmedetomidine dose-dependently inhibited thermal hyperalgesia in CFA-monarthritic rats ${ }^{[18]}$. Studies in mutant mice suggest that the anti-nociceptive effects of dexmedetomidine depend on $\mathrm{a}_{2} \mathrm{AR}$ located in the dorsal horn of the spinal cord ${ }^{[19]}$. Activation of $a_{2} A R$ can decrease the release of substance $P(S P)$ and calcitonin gene-related peptide (CGRP) from spinal cord slices or from cultured primary affer- 
ent neurons following noxious stimuli ${ }^{[20-23]}$. Also, it is reported that rat cultured astrocytes ${ }^{[24]}$ and microglial cells ${ }^{[25]}$ express mRNAs encoding $\mathrm{a}_{2} \mathrm{AR}$. Adrenergic agonist norepinephrine suppresses the expressions of mRNAs encoding proinflammatory cytokines ${ }^{[25]}$. However, whether dexmedetomidine in vivo influences spinal glial activation directly or indirectly under exaggerated pain conditions is unknown.

The aim of this study was to investigate whether repeated ip administration of dexmedetomidine could antagonize glial activation in the spinal dorsal horn, delay or attenuate thermal hyperalgesia in CFA-induced ankle joint monoarthritic rats.

\section{Materials and methods Animals}

Experiments were performed on adult male Sprague Dawley rats (Experimental Animal Center, Shanghai Medical College of Fudan University, China) weighing 180-200 g. Animals were housed in temperature-controlled $\left(22 \pm 2{ }^{\circ} \mathrm{C}\right)$ and lightcontrolled (12-h dark /12-h light cycle) room with free access to food and water. All experimental protocols and animal handling procedures were approved by Animal Care and Use Committee of Fudan University, and were consistent with the National Institutes of Health Guide for the Care and Use of Laboratory Animals. All efforts were made to minimize the number of animals used and their suffering.

\section{Drugs}

Dexmedetomidine hydrochloride (Jiangsu Hengrui Medicine $\mathrm{Co}, \mathrm{Ltd})$ was diluted in normal saline (NS, $0.9 \% \mathrm{NaCl})$. It was administered ip in a volume of $1 \mathrm{~mL} / \mathrm{kg}$ body weight. Control animals received equivalent volume of sterile NS.

\section{Induction of monoarthritis}

Monoarthritis (MA) was induced by an injection of complete Freund's adjuvant (CFA) into the unilateral ankle articular cavity. The rat was briefly anesthetized with isoflurane. The skin around the site of injection was sterilized with iodine tincture, then $75 \%$ alcohol. The left leg of the rat was held and the fossa of the lateral malleolus of the fibula was located. A 28-gauge needle was inserted vertically to penetrate the skin, and turned distally to insert into the articular cavity from the gap between the tibiofibular and tarsus bone until a distinct loss of resistance was felt. A volume of $50 \mu \mathrm{L}$ CFA was then injected. Sham MA control animals were similarly injected with sterile NS. Histopathological sections of the joints in CFA- and NS-injected rats were examined. A robust arthritislike inflammatory cells infiltrate was observed in CFA-injected joints (Figure 1).

\section{Hargreaves' test for thermal hyperalgesia}

After acclimation to the test chamber, thermal hyperalgesia was assessed by measuring the latency of paw withdrawal in response to a radiant heat source. Rats were housed individually into Plexiglas chambers on an elevated glass platform, under which a radiant heat source (model 336 combination unit, IITC/life Science Instruments, Woodland Hill, CA, USA)
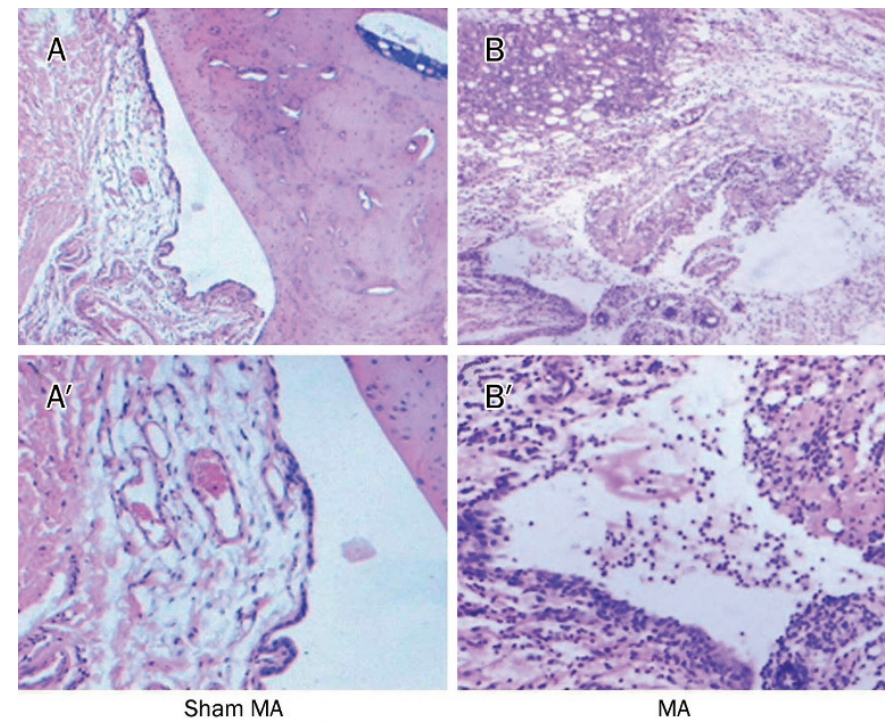

Figure 1. Histopathological sections of an ankle joint of a sham MA rat ( $A$ and $A^{\prime}$ ) and a CFA-induced MA rat ( $B$ and $\left.B^{\prime}\right)$. The joint of the rat with arthritis shows significantly joint destruction and robust arthritis-like inflammatory cell infiltration. $A$ and $B, 10 \times ; A^{\prime}$ and $B^{\prime}, \times 40$.

was applied to the plantar surface of the hind paw through the glass plate. The heat source was turned off when the rat lifted the foot, allowing the measurement of time from onset of radiant heat application to withdrawal of the rat's hindpaw. This time was defined as the paw withdrawal latency (PWL). The heat was maintained at a constant intensity, which produced a stable PWL of approximately 10-12 s in the absence of arthritis. A $20 \mathrm{~s}$ cutoff was used to prevent tissue damage. Both hindpaws were tested independently with $10 \mathrm{~min}$ interval between trials.

\section{Immunohistochemistry}

After defined survival times, rats were given an overdose of urethane $(2 \mathrm{~g} / \mathrm{kg}$, ip) and perfused intracardially with saline followed by $4 \%$ paraformaldehyde in $0.1 \mathrm{~mol} / \mathrm{L}$ phosphate buffer (PB, $\mathrm{pH} 7.4)$. The $\mathrm{L} 4 / 5$ segments of spinal cord were then removed, post-fixed in the same fixative for $4 \mathrm{~h}$ at $4{ }^{\circ} \mathrm{C}$, and immersed from $10 \%$ to $30 \%$ gradient sucrose in PB for $24-48 \mathrm{~h}$ at $4{ }^{\circ} \mathrm{C}$ for cryoprotection. Transverse spinal sections $(35 \mu \mathrm{m})$ were cut in a cryostat and processed for immunofluorescence. All the sections were blocked with $10 \%$ donkey serum in $0.01 \mathrm{~mol} / \mathrm{L}$ phosphate buffered saline (PBS, pH 7.4) with $0.3 \%$ Triton X-100 for $2 \mathrm{~h}$ at RT and incubated overnight at $4{ }^{\circ} \mathrm{C}$ with rabbit anti-Iba- 1 (1:2000, Woka), or mouse antiglial fibrillary acidic protein (GFAP, 1:2000, Sigma) primary antibody in PBS with 1\% normal donkey serum and $0.3 \%$ Triton X-100. Following three $10 \mathrm{~min}$ rinses in $0.01 \mathrm{~mol} / \mathrm{L}$ $\mathrm{PBS}$, the sections were incubated in fluorescein isothiocyanate (FITC)-conjugated donkey anti-mouse IgG (1:200, Jackson Immunolab) or rhodamine-conjugated donkey anti-rabbit IgG (1:200, Jackson Immunolab) for $120 \mathrm{~min}$ at $4{ }^{\circ} \mathrm{C}$, then washed in PBS. Omission of primary antibody served as negative 
control. All sections were coverslipped with a mixture of $50 \%$ glycerin in $0.01 \mathrm{~mol} / \mathrm{L} \mathrm{PBS}$, and then observed with a Leica fluorescence microscope, and images were captured with a CCD spot camera. Because the morphology of either microglia or astrocytes is complex and immunoreactive staining includes both cell bodies and their processes, cell counts may not sufficiently quantify activation. Therefore, the optical density of immunoreactive staining for Iba-1 or GFAP was measured with the Image J analysis system. For each animal, six sections of spinal L4-5 were randomly selected for quantitative evaluation. These corrected density values of six sections were averaged to provide a mean density for each animal.

\section{Western blot analysis}

After defined survival times, rats were killed by overdose of urethane. The lumbar spinal cords were rapidly removed. The dorsal horn was dissected using "open book" method. Briefly, the L4 and L5 spinal cord segments were first dissected according to the termination of the L4 and L5 dorsal roots. Then the spinal segments were split into a left (ipsilateral to MA side) and right (contralateral) half from the ventral midline. Finally, the left and right half were further cut into the dorsal and ventral horn at the level of the central canal. The dorsal horns were rapidly frozen in liquid nitrogen and stored at $-70{ }^{\circ} \mathrm{C}$ until further processing. In order to assess the development of arthritic pain, behavioral testing was performed immediately before sacrificed. Western blot analysis was conducted as described previously ${ }^{[26]}$, with slight modifications. Frozen spinal cords were directly homogenized in a lysis buffer $(12.5 \mathrm{~L} / \mathrm{mg}$ tissue) containing a cocktail of protease inhibitors and phenylmethanesulfonyl fluoride (PMSF, Sigma). Supernatant, after $10000 \times g$ centrifugation for $15 \mathrm{~min}$, was used for western blotting. Equal amount of protein $(20 \mathrm{~g})$ was loaded on each lane and separated by $10 \%$ SDS-PAGE for the GFAP detection. The resolved proteins were transferred onto PVDF membranes. The membranes were blocked in $10 \%$ nonfat dry milk in TBST with $0.1 \%$ Tween- 20 for $2 \mathrm{~h}$ at RT, and incubated overnight at $4{ }^{\circ} \mathrm{C}$ with mouse anti-GFAP (1:2000, Sigma) or mouse anti-GAPDH (1:10000, Sigma) primary antibody. The blots were incubated for $2 \mathrm{~h}$ at RT with horseradish peroxidase (HRP)-conjugate donkey anti-mouse secondary antibody (1:5000, Santa Cruze Biotechnology). Signals were finally visualized using enhanced chemiluminescence (ECL, Pierce) and exposed onto X-films for 1-10 min. All Western blot analysis was performed at least three times, and parallel results were obtained. X-ray films with blotting bands for each sample from different rats were scanned, and the density of band area was quantified with a method described by Zhuang et $a l^{[26]}$. The same size square was drawn around each band to measure the density and background near that band was subtracted. GAPDH expression was used as loading control for protein expression, and GFAP level was normalized against GAPDH level. The expression level for protein is an average of densities per band area from different treatment rats.

All the behavioral testing and quantification of immunohistochemical and immunoblotting experiments were performed blind with respect to treatments.

\section{Statistical analysis}

Data are given as the mean \pm SEM. Both pre-MA baseline and pre-drug treatment measures were analyzed by oneway analysis of variance (ANOVA). Post-drug time course measures for hyperalgesia were analyzed by two-way RM ANOVA (treatment $\times$ time) followed by Newman-Keuls post hoc test. Immunohistochemical and Western blot analysis was performed by Student's t-test when comparing two groups, or one-way ANOVA followed by Dunnett multiple comparisons when comparing more than two groups. $P<0.05$ was considered statistically significant.

\section{Experimental design}

Experiment 1 was designed to test the effects of repeated ip injection of dexmedetomidine on MA-induced thermal hyperalgesia. After baseline behavioral assessments, rats received an intra-articular injection of $50 \mu \mathrm{L}$ CFA or sterile NS (day 0). Repeated ip injection of dexmedetomidine $(2.5 \text { or } 10 \mu \mathrm{g} / \mathrm{kg})^{[18]}$ or vehicle (NS) were carried out once daily for 5 days after behavioral testing, with the first application $60 \mathrm{~min}$ before intra-articular injection of CFA. Hargreaves' test was performed from day 1 to 5 . Experiment 2 was designed to test the effects of repeated ip injection of dexmedetomidine on MAinduced glial activation in the lumbar spinal cord. Rats from experiment 1 were perfused on day 2 and day 5 after behavioral test, respectively, for immunohistochemical or Western blot processing with GFAP and Iba- 1 antibody. Sham MA rats received an intra-articular injection of sterile NS $(50 \mu \mathrm{L})$ and were killed at day 2 or day 5 after NS injection. Naïve rats could move freely in their home cages before they were killed.

\section{Results}

Effects of repeated ip dexmedetomidine on the development of thermal hypersensitivity in CFA-induced monoarthritic rats

Baseline measures of the paw withdrawal latency (PWL) to radiant heat stimulation did not differ in either hindpaw across groups. Unilateral intra-articular injection of CFA produced marked joint inflammation (edema and erythema) and thermal hyperalgesia in the ipsilateral paw to injection, which peaked at 1 day after CFA injection and showed little change during 5 day (Figure 2).

The effects of repeated ip injection of dexmedetomidine on PWLs to thermal stimulation were examined on each day after intra-articular injection of CFA. Ip injection of dexmedetomidine $(2.5$ or $10 \mu \mathrm{g} / \mathrm{kg}$ ) was repeatedly given once daily for 5 days with the first injection $60 \mathrm{~min}$ before intra-articular CFA. Multiple application of low dose dexmedetomidine (2.5 $\mu \mathrm{g} / \mathrm{kg}$ ) had no effect on MA-induced thermal hyperalgesia (Figure 2). However, at high dose of dexmedetomidine (10 $\mu \mathrm{g} / \mathrm{kg}$ ), single application before MA failed to prevent the development of thermal hyperalgesia that reliably occurred on day 1 post-MA. Following subsequent daily application, MA-induced ipsilateral hyperalgesia were significantly attenuated from day 2 to day 5 . One day after cessation of dexme- 


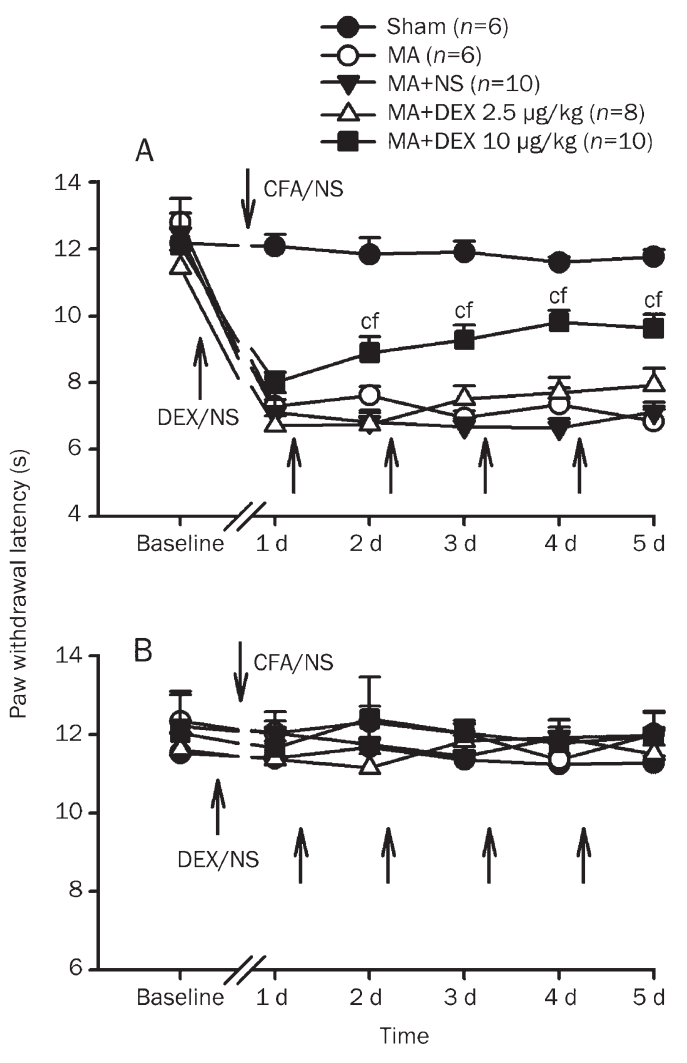

Figure 2. Effects of multiple intraperitoneal (ip) injections of dexmedetomidine (DEX, 2.5 and $10 \mu \mathrm{g} / \mathrm{kg}$ ) on paw withdrawal latencies (PWLs) of the hindpaws ipsilateral (A) and contralateral (B) to monoarthritis (MA) or sham MA. Repeated ip DEX or NS were given directly after behavioral test on days 1-4, with the first application 60 min before intra-articular injection of CFA or NS. ${ }^{c} P<0.01$ vs sham MA; ${ }^{f} P<0.01$ vs ip NS $(n=6-10)$.

detomidine injection, the PWLs were still reliably greater in dexmedetomidine-treated rats than that in vehicle-treated ones (Figure 2A). Two-way RM ANOVA analysis revealed significant effect of dexmedetomidine treatment $\left(F_{2,107}=23.98\right.$, $P<0.001)$ and interaction between dexmedetomidine treatment and time $\left(F_{5,107}=2.53, P=0.015\right)$. The contralateral PWLs were not obviously affected by repeated injection of dexmedetomidine (Figure 2B).

\section{Effects of repeated ip dexmedetomidine on MA-induced spinal microglial activation}

Our previous observation revealed early, robust and sustained microglial activation on the spinal dorsal horn during the acute ( 1 day), subacute ( 3 day), and chronic (10 day) phases of CFA-induced $\mathrm{MA}^{[7]}$. To examine whether repeatedly systemic administrations of dexmedetomidine affect MA-induced spinal microglial activation, Iba-1 (microglial marker) immunoreactivity was assessed on the lumbar spinal cord in the present study. As expected, unilateral intra-articular injection of CFA produced a robust increase in the expression for Iba-1 immunoreactivity (Iba-1-IR) on both sides of the lumbar spinal dorsal horn with the more prominent increase being on the ipsilateral spinal cord on day 5 post-MA (Figure 3). Activated microglia showed shrunk processes, hypertrophic cell bodies and intense Iba- 1 immunoreactivity. Repeated ip injections of dexmedetomidine $(10 \mu \mathrm{g} / \mathrm{kg})$ caused a trend to decrease in Iba-1 immunoreactivity on both sides of the spinal cord by day 5 post-MA, although that did not reach statistical significance (Figure 3B).

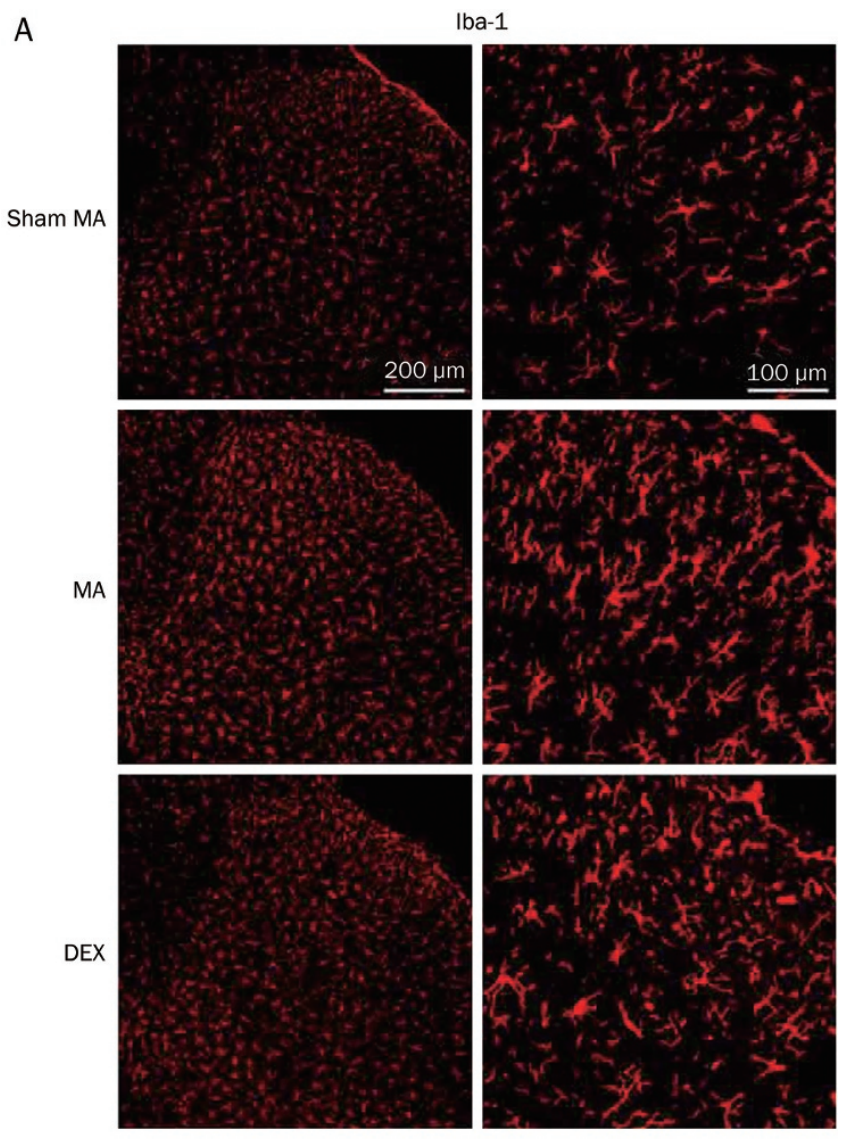

B

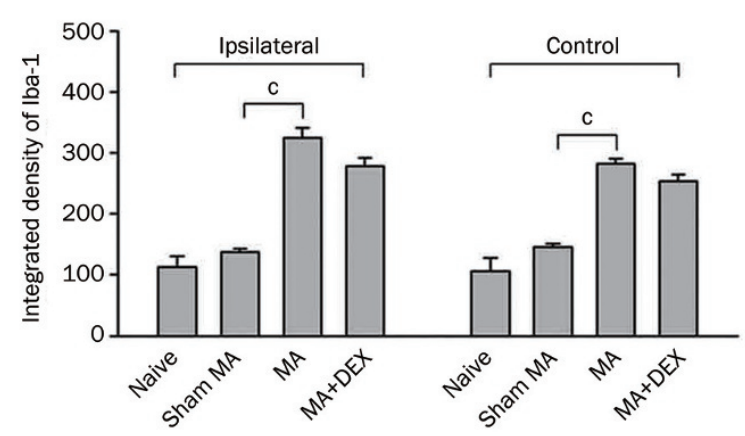

Figure 3. Effects of repeated ip injections of dexmedetomidine (DEX) on increase in Iba-1 (a microglial marker) expression in the spinal dorsal horn induced by monoarthritis (MA). (A) Immunohistochemistry for lba-1 in the ipsilateral spinal transverse sections on day 5 after sham MA, MA, and MA with multiple DEX-treatment. (B) Quantification of Iba-1 expression on both sides of the spinal dorsal horn. ${ }^{\mathrm{c}} P<0.01(n=4)$. 
Effects of repeated ip dexmedetomidine on MA-induced spinal astrocytic activation.

To examine whether repeatedly systemic applications of dexmedetomidine affect MA-induced spinal astrocytic activation, GFAP (astrocytic marker) immunoreactivity and immunoblotting was assessed on the lumbar spinal cord in naive, sham MA, MA and dexmedetomidine-treated rats. As previous report ${ }^{[8]}$, significant elevation of GFAP expression was observed in both sides of the spinal dorsal horn on day 2 and day 5 after MA (Figure 4 and 5). Twice administered dexmedetomidine $(10 \mu \mathrm{g} / \mathrm{kg})$ did not decrease GFAP level in the spinal dorsal horn on day 2 after MA (Figure 4A-4C). Following subsequent daily application, dexmedetomidine $(10 \mu \mathrm{g} / \mathrm{kg})$ significantly suppressed GFAP expression by day 5 (Figure 4D-4F and 5). Repeated ip injections of $2.5 \mu \mathrm{g} / \mathrm{kg}$ dexmedetomidine did not suppress MA-induced upregulation of GFAP on day 5 (Figure $4 \mathrm{E}$ and $4 \mathrm{~F}$ ).

\section{Discussion}

A new finding of the present study is that repeated administered (ip) of dexmedetomidine, a highly specific potent and selective $\mathrm{a}_{2} \mathrm{AR}$ agonist, markedly suppressed ankle joint inflammation-induced glial activation on the spinal dorsal horn, which was associated with its ability to attenuate thermal hyperalgesia of the monoarthritic rats.

Dexmedetomidine, approved by the Food and Drug Administration in 1999, has been used in humans as a medication for analgesia and sedation in the intensive care unit (ICU) ${ }^{[27]}$. In experimental animal study, it has been shown to exert dosedependent analgesic potential in inflammatory ${ }^{[15,18]}$, neuropathic $^{[16]}$ and postoperative pain ${ }^{[17]}$. In agreement with the previous studies, the present study further demonstrated repeated ip dexmedetomidine, initiating from early phase of inflammation, significantly suppressed MA-induced thermal hyperalgesia and spinal glial activation, especially astrocytes. The $\mathrm{a}_{2} \mathrm{AR}$ is located diffusely in the nervous system including primary afferents, spinal dorsal horn neurons and brainstem $^{[28]}$. Systemic or intrathecal application of $a_{2} A R$ agonists including dexmedetomidine and clonidine produces significant anti-nociceptive effect, suggesting all the levels of supraspinal, spinal and peripheral $\mathrm{a}_{2} \mathrm{AR}$ might be involved in the anti-nociception ${ }^{[29-31]}$. However, a study from Basbaum's laboratory showed that systemic delivery of dexmedetomidine blocked nerve injury-induced thermal and mechanical allodynia in wild-type, but not $\mathrm{a}_{2 \mathrm{~A}}$-AR mutant, mice; and the effect of dexmedetomidine was reversed by intrathecal, but not systemic, injection of the $\mathrm{a}_{2} \mathrm{AR}$ antagonist RS 42206, strongly suggesting that the spinal $a_{2 A}-A R$ is essential for the antinociceptive effects of dexmedetomidine ${ }^{[19]}$.

Glial cells were first considered as contributing to exaggerated "pain" by Garrison et $a l^{[32]}$. They found that manipulation that created exaggerated "pain" also activated astrocytes, and the drug that blocked exaggerated "pain" also blocked astrocytes activation. Since then, glial (both microglia and astrocytes) activation has been observed at the lumbar spinal cord in various animal models of pathological pain ${ }^{[2,7,10,11,33,34]}$.
A

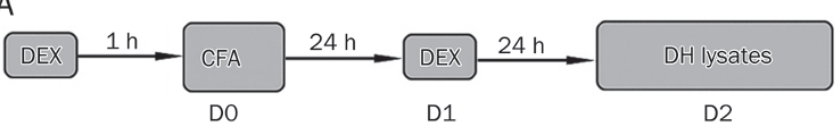

B

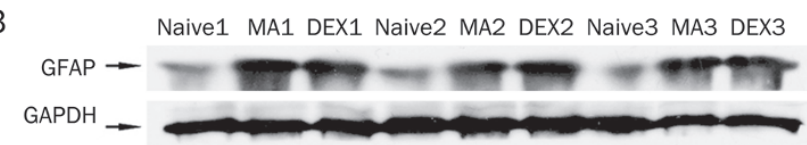

C

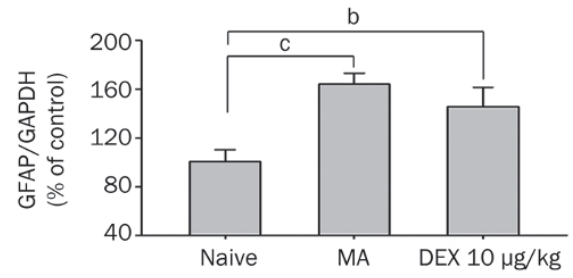

D

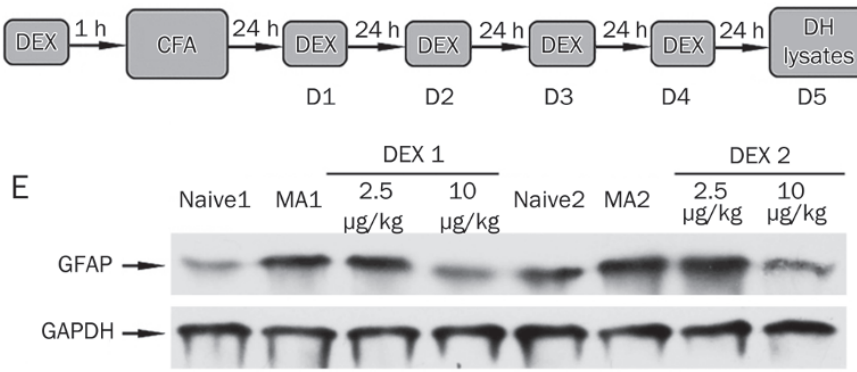

$\mathrm{F}$

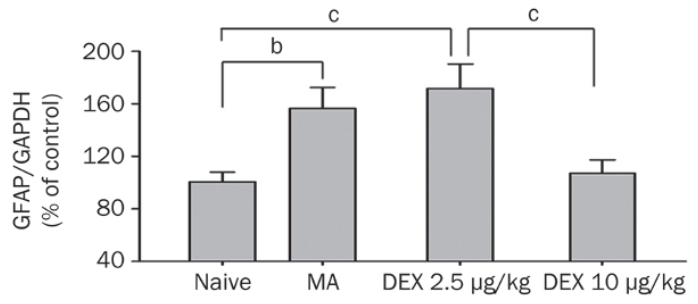

Figure 4. Western blot for GFAP and GAPDH from the spinal dorsal horn of naïve, MA, and dexmedetomidine (DEX)-treated rats. A-C, Effect of twice ip DEX (10 $\mathrm{\mu g} / \mathrm{kg})$ on increase in GFAP level in the spinal dorsal horn induced by monoarthritis (MA) on day 2 after MA. D-F, Effect of repeated ip injections of DEX ( 2.5 and $10 \mu \mathrm{g} / \mathrm{kg}$ ) on increase in GFAP level in the spinal dorsal horn induced by MA on day 5 after MA. A and D show protocol of subsequent studies; $B$ and $E$ show examples of Western blot analysis; $C$ and $F$ show quantification of GFAP protein levels in different treatments. Since not detectable differences in the GFAP expression level between both sides of the spinal dorsal horn, two sides of the spinal cord were pooled together in this experiment. GAPDH served as loading control. ${ }^{\mathrm{b}} P<0.05,{ }^{\mathrm{c}} P<0.01(n=4)$.

Despite some conflicting reports ${ }^{[35-37]}$, most evidence showed that spinal microglia and astrocytes were activated by subcutaneous injection of $\mathrm{CFA}^{[6]}$, phospholipase $\mathrm{A}^{[38]}{ }^{\text {, snake }}$ venom $^{[39]}$, formalin ${ }^{[28,33,40-42]}$ and zymosan ${ }^{[34]}$. The recent studies from our laboratory further demonstrated that a robust microglial activation on the spinal dorsal horn was observed 
A

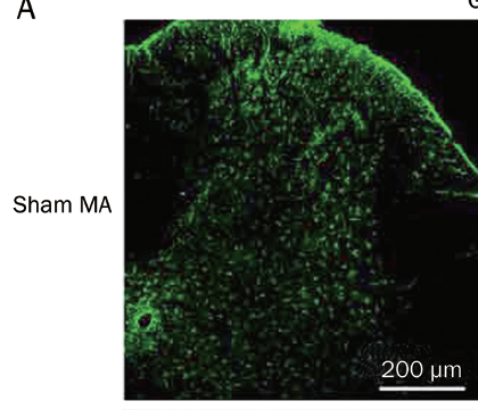

GFAP
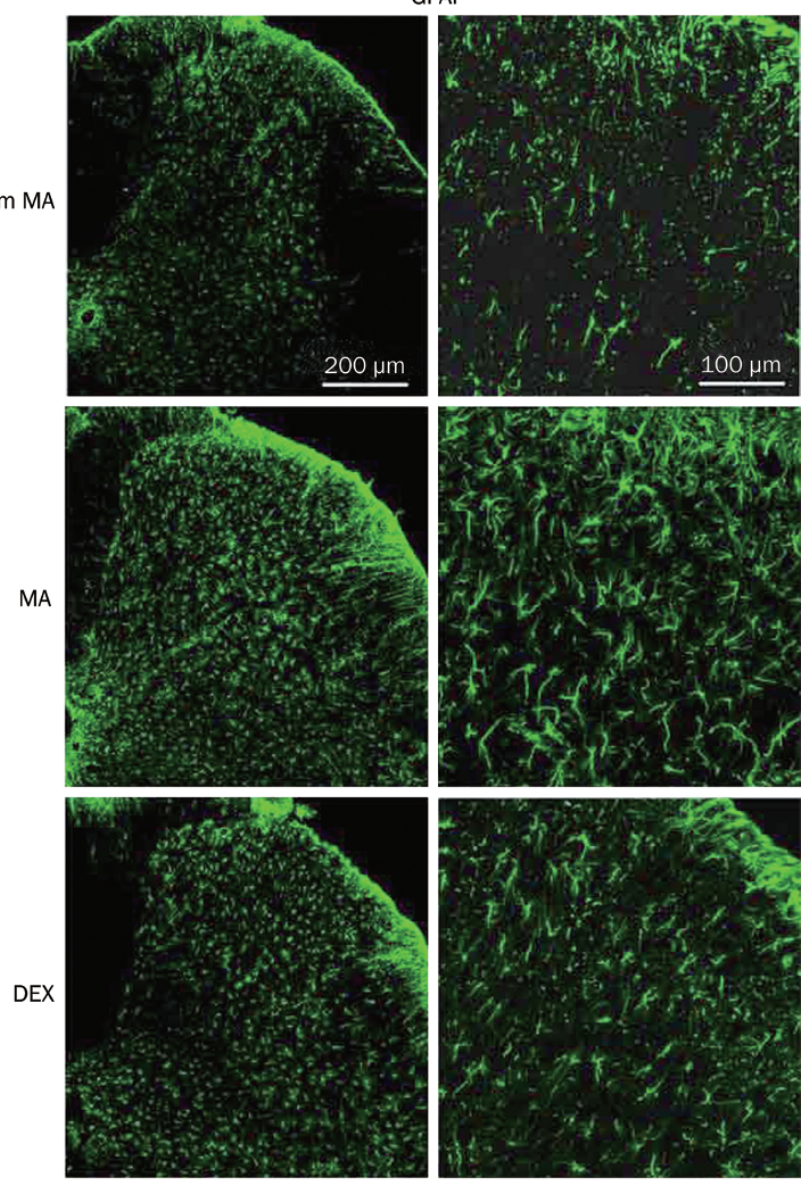

B

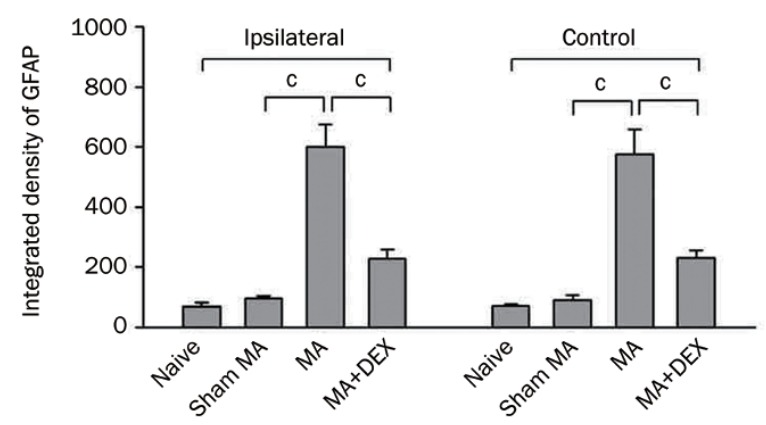

Figure 5. Effects of repeated ip injections of dexmedetomidine (DEX) on increase in GFAP (a astrocytic marker) expression in the spinal dorsal horn induced by monoarthritis (MA). (A) Immunohistochemistry for GFAP in the ipsilateral spinal transverse sections on day 5 after sham MA, MA, and MA with multiple DEX-treatment. (B) Quantification of GFAP expression on both sides of the spinal dorsal horn. ${ }^{c} P<0.01(n=4)$.

at days 1, 3, and 10 after intraarticular injection of CFA, and significant astrocytic activation occurred on day 3 and $10^{[7-9]}$. Consistently, the present results showed robust activation of both microglia and astrocytes on day 5 after MA. Different from peripheral neuropathy that primarily results in spinal glial activation on ipsilateral to the injury, peripheral inflam- mation usually produces bilateral glial activation in the spinal $\operatorname{cord}^{[2,34]}$. We could not give a clear explanation of this phenomenon at the present, one supposed explanation is that the generalized bilateral glial response to inflammation results from systemic or perivascular signals ${ }^{[34,43]}$. It has been demonstrated that in status of inflammatory pain, partial opening of the tight junction permeability between the endothelial cells of the capillaries, increased blood-brain barrier permeability ${ }^{[44]}$.

Activated glia produce and release various algesic substances, such as pro-inflammatory cytokines and other mediators, which can enhance pain transmission ${ }^{[4]}$. Spinal glia express an array receptors including NMDA, AMPA/KA, NK-1, CGRP, etc ${ }^{[33,45-47]}$. A variety of classical "pain" neurotransmitters/neuromodulators including glutamate, ATP, SP, CGRP, nitric oxide (NO) and prostaglandins (PGs) can cause spinal glial activation. It has been shown that activation of $\mathrm{a}_{2} \mathrm{AR}$ reduces capsaicin, potassium and noxious stimulusevoked release of SP and CGRP ${ }^{[20-23]}$, as well as excitatory amino acids ${ }^{[48]}$ from spinal cord slices or from cultured primary afferent neurons. Thus, one of major mechanisms of dexmedetomidine suppressing MA-induced spinal glial activation might be to diminish the release of some "pain" neurotransmitters/neuromodulators (such as excitatory amino acids and neuropeptides) from primary afferent terminals and spinal nociceptive neurons via $a_{2} A R$. The present results that twice dexmedetomidine with the first application before MA significantly suppressed MA-induced thermal hyperalgesia, but failed to block GFAP upregulation in the spinal cord on day 2 after MA suggest an indirect or secondary inhibitory effect of dexmedetomidine on astrocytic activation. In addition, it was reported that $\mathrm{a}_{2} \mathrm{AR}$ antagonist yohimbine reduce chronic morphine administration-induced GFAP upregulation in the frontal cortex, nucleus acuumbens, ventral tegmental area, locus ceruleus and nucleus of the solitary, which was consistent with an indirect effect of $\mathrm{a}_{2} \mathrm{AR}$ modulators on

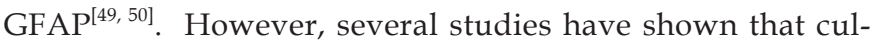
tured astrocytic or microglial cells express $\mathrm{a}_{2} \mathrm{AR}$ or mRNAs encoding $\mathrm{a}_{2} \mathrm{AR}^{[24,25]}$. In the hippocampus in vivo, it was also observed that numerous astrocytic processes contained immunoreactivity for $\mathrm{a}_{2 \mathrm{~A}}-\mathrm{AR}{ }^{[51]}$. Thus, a direct inhibitory effect of dexmedetomidine on spinal glial cells may not be ruled out completely, although $\mathrm{a}_{2} \mathrm{ARs}$ were not detected in spinal astrocytes by an early study ${ }^{[52]}$.

In summary, the present study demonstrated for the first time that systemic administration of dexmedetomidine markedly suppressed ankle joint inflammation-induced glial activation on the spinal dorsal horn, which is possibly associated with its antihyperalgesia in MA rats. The $\mathrm{a}_{2}$ ARs located in the primary afferent terminals and nociceptive neurons in the spinal dorsal horn and brainstem might be implied in the mechanisms of systemic dexmedetomidine suppressing MA-induced spinal glial activation.

\section{Acknowledgements}

This work was supported by National Natural Science Fundation of China (NSFC, 30870835, and 30821002) and 
National Basic Research Program of China (2007CB512303, 2007CB512502, and 2006CB500807).

\section{Author contribution}

Bo XU and Wei-shi ZHANG carried out most of the behavioral testing and immunohistochemical experiments. Jia-le YANG performed parts of immunohistochemical experiments. Hua $\mathrm{XU}$, Xiao-ming DENG, and Yu-qiu ZHANG conceived the study, designed the experiments, participated in parts of data analysis and helped to draft the manuscript.

\section{References}

1 Schaible HG, Ebersberger A, Von Banchet GS. Mechanisms of pain in arthritis. Ann N Y Acad Sci 2002; 966: 343-54.

2 Cao $\mathrm{H}$, Zhang YQ. Spinal glial activation contributes to pathological pain states. Neurosci Biobehav Rev 2008; 32: 972-83.

3 Deleo JA, Yezierski RP. The role of neuroinflammation and neuroimmune activation in persistent pain. Pain 2001: 90; 1-6.

4 Watkins LR, Milligan ED, Maier SF. Glial activation: a driving force for pathological pain. Trends Neurosci 2001; 24: 450-5.

5 Hua XY, Svensson Cl, Matsui T, Fitzsimmons B, Yaksh TL, Webb M. Intrathecal minocycline attenuates peripheral inflammation-induced hyperalgesia by inhibiting p38 MAPK in spinal microglia. Eur J Neurosci 2005; 22: 2431-40.

6 Raghavendra V, Tanga FY, DeLeo JA. Complete Freunds adjuvantinduced peripheral inflammation evokes glial activation and proinflammatory cytokine expression in the CNS. Eur J Neurosci 2004; 20: 467-73.

7 Sun S, Mao Ying QL, Cao H, Li TT, Han M, Pang HL, et al. Is functional state of spinal microglia involved in the anti-allodynic and antihyperalgesic effects of electroacupuncture in rat model of monoarthritis? Neurobiol Dis 2007; 26: 558-68.

8 Sun S, Cao H, HanM, Li TT, Zhao ZQ, Zhang YQ. Evidence for suppression of electroacupuncture on spinal glial activation and behavioral hypersensitivity in a rat model of monoarthritis. Brain Res Bull 2008; 75: 83-93.

9 Sun S, Cao H, Han M, Li TT, Pan HL, Zhao ZQ, et al. New evidence for the involvement of spinal fractalkine receptor in pain facilitation and spinal glial activation in rat model of monoarthritis. Pain 2007; 129: 64-75.

10 Ledeboer A, Sloane EM, Milligan ED, Frank MG, Mahony JH, Maier SF, et al. Minocycline attenuates mechanical allodynia and proinflammatory cytokine expression in rat models of pain facilitation. Pain 2005; 115: 71-83.

11 Milligan ED, Twining C, Chacur M, Biedenkapp J, O'Connor K, Poole S, et al. Spinal glia and proinflammatory cytokines mediate mirror-image neuropathic pain in rats. J Neurosci 2003; 23: 1026-40.

12 Sun S, Chen WL, Wang PF, Zhao ZQ, Zhang YQ. Disruption of glial function enhances electroacupuncture analgesia in arthritic rats. Exp Neurol 2006: 198; 294-302.

13 Al-Metwalli RR, Mowafi HA, Ismail SA, Siddiqui AK, Al-Ghamdi AM, Shafi MA, et al. Effect of intra-articular dexmedetomidine on postoperative analgesia after arthroscopic knee surgery. Br J Anaesth 2008; 101: 395-9.

14 Jaakola ML, Salonen M, Lehtinen R, Scheinin H. The analgesic action of dexmedetomidine - a novel alpha 2-adrenoceptor agonist - in healthy volunteers. Pain 1991; 46: 281-5.

$15 \mathrm{Xu}$ M, Kontinen VK, Kalso E. Effects of radolmidine, a novel alpha2adrenergic agonist compared with dexmedetomidine in different pain models in the rat. Anesthesiology 2000; 93: 473-81.
16 Guneli E, Karabay Yavasoglu NU, Apaydin S, Uyar M, Uyar M. Analysis of the antinociceptive effect of systemic administration of tramadol and dexmedetomidine combination on rat models of acute and neuropathic pain. Pharmacol Biochem Behav 2007; 88: 9-17.

17 Shimode N, Fukuoka T, Tanimoto M, Tashiro C, Tokunaga A, Noguchi K. The effects of dexmedetomidine and halothane on Fos expression in the spinal dorsal horn using a rat postoperative pain model. Neurosci Lett 2003; 343: 45-8.

18 Zhang WS, Xu H, Xu B, Sun S, Deng XM, Zhang YQ. Antihyperalgesic effect of systemic dexmedetomidine and gabapentin in a rat model of monoarthritis. Brain Res 2009; 1264: 57-66.

19 Malmberg AB, Hedley LR, Jasper JR, Hunter JC, Basbaum Al. Contribution of alpha(2) receptor subtypes to nerve injury-induced pain and its regulation by dexmedetomidine. Br J Pharmacol 2001; 132: 1827-36.

20 Bourgoin S, Pohl M, Mauborgne A, Benoliel JJ, Collin E, Hamon M, et al. Monoaminergic control of the release of calcitonin gene related peptide- and substance P-like materials from rat spinal cord slices. Neuropharmacology 1993; 32: 633-40.

21 Holz GG4th, Kream RM, Spiegel A, Dunlap K. G proteins couple alphaadrenergic and GABAb receptors to inhibition of peptide secretion from peripheral sensory neurons. J Neurosci 1989; 9: 657-66.

22 Pang IH, Vasko MR. Effect of depletion of spinal cord norepinephrine on morphine-induced antinociception. Brain Res 1986; 371: 171-6.

23 Takano Y, Yaksh TL. Chronic spinal infusion of dexmedetomidine, ST91 and clonidine: spinal alpha2 adrenoceptor subtypes and intrinsic activity. J Pharmacol Exp Ther 1993; 264: 327-35.

24 Enkvist MO, Hämäläinen $\mathrm{H}$, Jansson CC, Kukkonen JP, Hautala R, Courtney MJ, Akerman KE. Coupling of astroglial alpha 2-adrenoreceptors to second messenger pathways. J Neurochem. 1996; 66: 2394-401.

25 Mori K, Ozaki E, Zhang B, Yang L, Yokoyama A, Takeda I, et al. Effects of norepinephrine on rat cultured microglial cells that express alpha1, alpha2, beta1 and beta2 adrenergic receptors. Neuropharmacology 2002; 43: 1026-34.

26 Zhuang ZY, Gerner P, Woolf CJ, Ji RR. ERK is sequentially activated in neurons, microglia, and astrocytes by spinal nerve ligation and contributes to mechanical allodynia in this neuropathic pain model. Pain 2005; 114: 149-59.

27 Coursin DB, Coursin DB, Maccioli GA. Dexmedetomidine. Curr Opin Crit Care 2001; 7: 221-26.

28 Unnerstall JR, Kopajtic TA, Kuhar MJ. Distribution of alpha 2 agonist binding sites in the rat and human central nervous system: analysis of some functional, anatomic correlates of the pharmacologic effects of clonidine and related adrenergic agents. Brain Res 1984; 319: 69-101.

29 Guo TZ, Jiang JY, Buttermann AE, Maze M. Dexmedetomidine injection into the locus ceruleus produces antinociception. Anesthesiology 1996; 84: 873-81.

30 Ishii H, Kohno T, Yamakura T, Ikoma M, Baba H. Action of dexmedetomidine on the substantia gelatinosa neurons of the rat spinal cord. Eur J Neurosci 2008; 27: 3182-90.

31 Nakamura M, Ferreira SH. Peripheral analgesic action of clonidine: mediation by release of endogenous enkephalin-like substances. Eur J pharmacol 1988; 146: 223-8.

32 Garrison CJ, Dougherty PM, Carlton SM. GFAP expression in lumbar spinal cord of naive and neuropathic rats treated with MK-801. Exp Neurol 1994; 129: 237-43.

33 Raghavendra V, Rutkowski MD, DeLeo JA. The role of spinal neuroimmune activation in morphine tolerance/hyperalgesia in neuropathic and sham-operated rats. J Neurosci 2002; 22: 9980-9. 
34 Sweitzer SM, Colburn RW, Rutkowski M, DeLeo JA. Acute peripheral inflammation induces moderate glial activation and spinal IL-1beta expression that correlates with pain behavior in the rat. Brain Res 1999; 829: 209-21.

35 Clark AK, Gentry C, Bradbury EJ, McMahon SB, Malcangio M. Role of spinal microglia in rat models of peripheral nerve injury and inflammation. Eur J Pain 2007; 11: 223-30.

36 Lin T, Li K, Zhang FY, Zhang ZK, Light AR, Fu KY. Dissociation of spinal microglia morphological activation and peripheral inflammation in inflammatory pain models. J Neuroimmunol 2007; 192: 40-8.

37 Lindia JA, McGowan E, Jochnowitz N, Abbadie C. Induction of CX3CL1 expression in astrocytes and CX3CR1 in microglia in the spinal cord of a rat model of neuropathic pain. J Pain 2005; 6: 434-8.

38 Raghavendra V, Agrewala JN, Kulkarni SK. Melatonin reversal of lipopolysaccharides-induced thermal and behavioral hyperalgesia in mice. Eur J Pharmacol 2000; 395: 15-21.

39 Chacur M, Gutierrez JM, Milligan ED, Wieseler-Frank J, Britto LRG, Maier SF, et al. Snake venom components enhance pain upon subcutaneous injection: an initial examination of spinal cord mediators. Pain 2004; 111: 65-76.

40 Guo LH, Trautmann K, Schluesener HJ. Expression of P2X4 receptor by lesional activated microglia during formalin-induced inflammatory pain. J Neuroimmunol 2005; 163: 120-7.

41 Lan L, Yuan H, Duan L, Cao R, Gao B, Shen J, et al. Blocking the glial function suppresses subcutaneous formalin-induced nociceptive behavior in the rat. Neurosci Res 2007; 57: 112-9.

42 Watkins LR, Martin D, Ulrich P, Tracey KJ, Maier SF. Evidence for the involvement of spinal cord glia in subcutaneous formalin induced hyperalgesia in the rat. Pain 1997; 71: 225-35.

43 Elmquist JK, Scammell TE, Saper CB. Mechanisms of CNS response to systemic immune challenge: the febrile response. Trends Neurosci.
1997; 20: 565-70.

44 Huber JD, Witt KA, Hom S, Egleton RD, Mark KS, Davis TP. Inflammatory pain alters blood-brain barrier permeability and tight junctional protein expression. Am J Physiol Heart Circ Physiol 2001; 280: H1241-8.

45 Araque A, Parpura V, Sanzgiri RP, Haydon PG. Tripartite synapses: glia, the unacknowledged partner. Trends Neurosci 1999; 22: 208-15.

46 Marriott I. The role of tachykinins in central nervous system inflammatory responses. Frontiers in Bioscience 2004; 9: 2153-65.

47 Tsuda M, Shigemoto-Mogami Y, Koizumi S, Mizokoshi A, Kohsaka S, Salter MW, et al. P2X4 receptors induced in spinal microglia gate tactile allodynia after nerve injury. Nature 2003; 424: 778-83.

$48 \mathrm{Li} \mathrm{X}$, Eisenach JC. alpha2A-adrenoceptor stimulation reduces capsaicin-induced glutamate release from spinal cord synaptosomes. J Pharmacol Exp Ther 2001; 299: 939-44.

49 Garrido E, Perez-Garcia C, Alguacil LF, Diez-Fernandez C. The alpha 2-adrenoceptor antagonist yohimbine reduces glial fibrillary acidic protein upregulation induced by chronic morphine administration. Neurosci Lett 2005; 383: 141-4.

50 Alonso E, Garrido E, Diez-Fernandez C, Perez-Garcia C. Herradon G, Ezquerra $\mathrm{L}$, et al. Yohimbine prevents morphine-induced changes of glial fibrillary acidic protein in brainstem and alpha2-adrenoceptor gene expression in hippocampus. Neurosci Lett 2007; 412; 163-7.

51 Milner TA, Lee A, Aicher SA, Rosin DL. Hippocampal alpha2a-adrenergic receptors are located predominantly presynaptically but are also found postsynaptically and in selective astrocytes. J Comp Neurol. 1998; 395: 310-27.

52 Stone LS, Broberger C, Vulchanova L, Wilcox GL, Hokfelt T, Riedl MS, et al. Differential distribution of alpha2A and alpha2C adrenergic receptor. Immunoreactivity in the rat spinal cord. J Neurosci 1998; 18: 5928-37. 